\title{
Perspectives
}

\section{Biology of Mammalian Fertilization: Role of the Zona Pellucida}

\author{
Jurrien Dean \\ Laboratory of Cellular and Developmental Biology, National Institute of Diabetes and Digestive and Kidney Diseases, \\ National Institutes of Health, Bethesda, Maryland 20892
}

\section{Introduction}

In mammals, a series of carefully orchestrated events culminate in the fusion of a sperm and egg to form a one-cell zygote, the obligatory precursor of all cells in the embryo. The overall rate of fertilization in humans has contributed to sustained increases in the world population and added urgency to the need to develop new, effective contraceptive agents. For some, however, the success rate is considerably lower, and there are millions of infertile couples in the United States. Dramatic advances in reproductive biology have provided some relief for these individuals through the development of in vitro fertilization techniques. Further progress requires additional understanding of the molecular basis for normal fertilization and our willingness to exploit this knowledge to facilitate desired pregnancies or prevent unwanted ones. These joint desires to have greater control over our reproductive destiny has led to an increased interest in the macromolecules involved in fertilization.

The zona pellucida, an extracellular matrix surrounding the female gamete, contains the primary and secondary sperm receptors and plays a pivotal role in sperm-egg interactions. Although millions of sperm are deposited in the female reproductive tract, fewer than 100 approach the ovulated egg in the oviduct, and normally only one successfully penetrates through the zona pellucida to fuse with the plasma membrane of the ovum. Immediately following fertilization, the plasma membrane and zona pellucida are modified to prevent polyspermy and, perhaps, to provide additional resiliency to the zona so that it can protect the preimplantation embryo. Interactions between the sperm and egg appear to be relatively species-specific and research has focused on the male and female macromolecules involved. This perspective will concentrate on recent advances in our knowledge of the zona pellucida genes and proteins of the mouse, an experimental animal that has provided much of our current understanding of the molecular details of fertilization.

\section{Fertilization: a precis}

After ejaculation into the female reproductive tract, sperm undergo a poorly understood maturation process (capacitation)

Address reprint requests to Dr. Jurrien Dean, Laboratory of Cellular and Developmental Biology, National Institute of Diabetes and Digestive and Kidney Diseases, Building 6, Room B1-26, National Institutes of Health, Bethesda, MD 20892.

Received for publication 31 December 1991 and in revised form 21 January 1992.

The Journal of Clinical Investigation, Inc.

Volume 89, April 1992, 1055-1059 before approaching the ovulated egg in the oviduct (Fig. $1 A$ ). Motile sperm pass through the enveloping cumulus oophorus, which is composed of a glycosylaminoglycan matrix and cumulus cells. They then bind to the zona pellucida that surrounds the mammalian egg $(1,2)$. The mouse and human zonae pellucidae are composed of three major glycoproteins, ZP1, ZP2, and ZP3. Solubilized zonae pellucidae from unfertilized mouse eggs (but not from two-cell embryos) can inhibit sperm binding to ovulated eggs (3). This sperm-receptor activity of the zona has been ascribed to a class of 3.9-kD O-linked oligosaccharides on ZP3 (4). ZP2 has been implicated as a secondary sperm receptor that binds sperm only after the induction of the sperm acrosome reaction (5).

The acrosome is a membrane-bound organelle anteriorly located in the head of the sperm. It has been proposed that the binding of sperm to ZP3 induces a signal transduction across the sperm membrane by aggregating a sperm-specific $95-\mathrm{kD}$ protein with tyrosine kinase activity $(6,7)$. This leads to the acrosome reaction (8) in which the sperm plasma membrane fuses with the outer acrosomal membrane, resulting in the exocytosis of the acrosomal contents (Fig. $1 B$ ). The lytic enzymes (e.g., acrosin, glycosidases) that are released, as well as some that remain associated with the inner acrosomal membrane, such as acrosin, appear to facilitate passage of the motile sperm through the zona pellucida.

A number of sperm surface macromolecules have been proposed as zona adhesion molecules, e.g., serine proteinases and $\beta$-1,4-galactosyltransferase. More recent candidates include a $95,000-\mathrm{D}$ protein with tyrosine kinase activity and a 56,000-D protein isolated by its ability to bind ZP3 (for review see [9]). It is likely that more than one interaction is responsible for the binding of sperm to the zona, both initially and during penetration. Some of the sperm ligands present on the surface of the inner acrosomal membrane (e.g., proacrosin and PH-20) may become available for zona binding only after the sperm acrosome reaction. These latter molecules may be particularly important for successful penetration of the zona pellucida.

Immediately after fertilization there are two major changes that prevent polyspermy: a rapid electrical depolarization of the egg plasma membrane that blocks additional sperm in the perivitelline space from fusing with the egg (10), and biochemical modifications of the zona pellucida. These latter changes occur secondarily to the fusion of cytoplasmic cortical granules with the egg plasma membrane, and the subsequent discharge of the granules' enzymatic contents into the perivitelline space. The release of proteinases and glycosidases modifies the zona pellucida (zona reaction), resulting in a block to additional sperm binding and inhibition of zona-bound sperm penetration $(1,10)$. Both $\mathrm{ZP} 2$ and $\mathrm{ZP} 3$ are modified by the zona reaction: ZP2 undergoes a proteolytic cleavage associated with the 

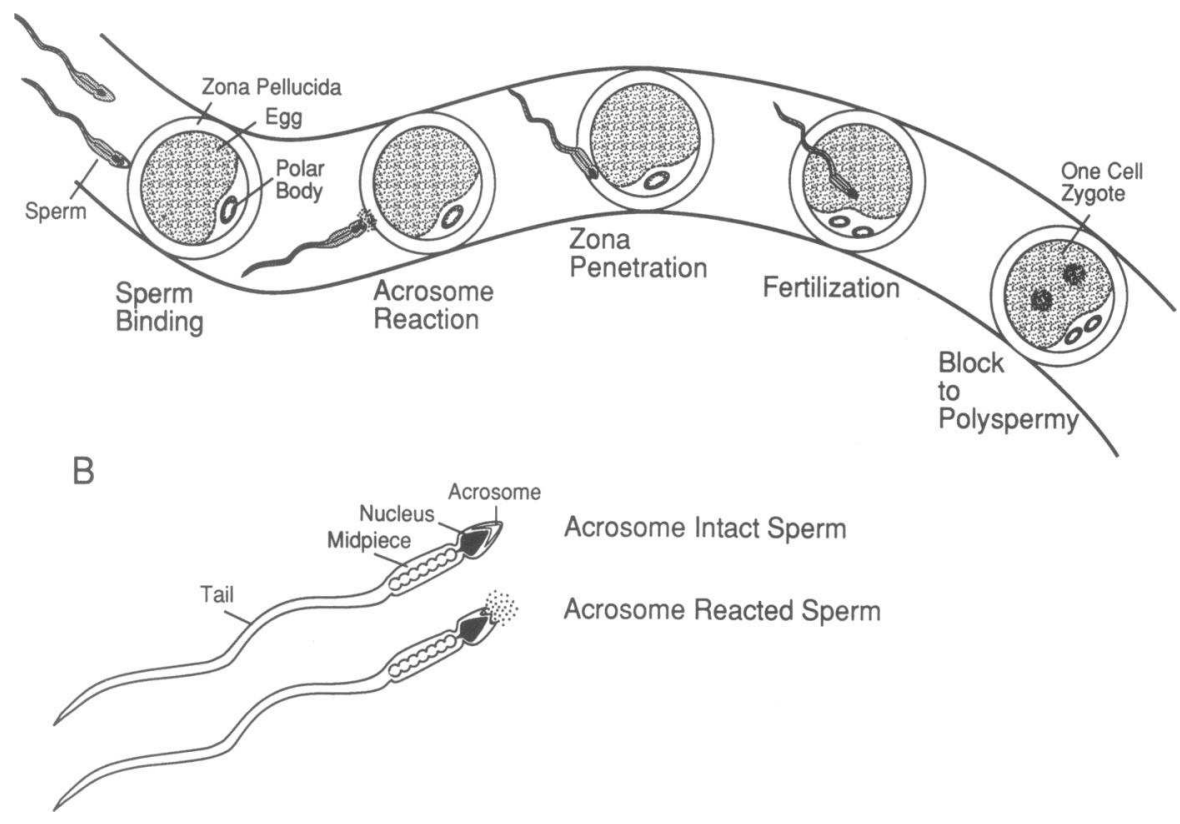

Figure 1. Mammalian fertilization. (A) The binding of sperm to the zona pellucida surrounding the ovulated egg induces the sperm acrosome reaction. The release of lytic enzymes from the acrosome and the forward motility of the sperm permit penetration of the zona pellucida. After fusion with the egg's plasma membrane, the sperm enters the cytoplasm and forms the male pronucleus of the one cell zygote. The female pronucleus, formed at the same time, contains the female haploid genome. Following fertilization, the zona pellucida is biochemically modified to prevent additional sperm from binding or penetrating the zona. (B) The acrosome, a lysosomallike structure on the anterior head of sperm, contains an inner and outer membrane that fuse during the acrosome reaction. This results in the release of lytic enzymes that are important for the penetration of the zona pellucida by the sperm.

block to polyspermy (11), and ZP3 loses both its ability to induce the acrosome reaction and its sperm receptor activity $(8,12)$.

\section{The zona pellucida proteins}

The zona pellucida proteins are synthesized, glycosylated, and sulfated in oocytes before their secretion to form the zona matrix that surrounds the growing oocyte, ovulated egg and early embryo.

Structure. Mouse ZP1 (185-200 kD) is a disulfide-linked dimer which has yet to be characterized in molecular detail. The primary amino acid sequences of mouse ZP2 (120-140 $\mathrm{kD})$ and $\mathrm{ZP} 3(83 \mathrm{kD})$ have been deduced from the nucleic acid sequence of their cognate genes (see below). Although the peptide backbones of the human ZP2 and ZP3 are similar in length to those of their mouse homologues (see below), the mature human proteins are smaller, ZP1 (90-110 kD), ZP2 (64-76 $\mathrm{kD}$ ), and ZP3 (57-73 kD) (13), presumably due to differences in glycosylation. Recent experiments with immunological probes have identified two isoforms of human $\mathrm{ZP} 3, \mathrm{ZP} 3_{\mathrm{H}}$ and $Z P 3_{L}$ (14), but whether these represent different posttranscriptional modifications of a single ZP3 gene product or the presence of an additional gene remains to be determined.

Biosynthesis. There is no zona pellucida surrounding resting mouse oocytes, and zona protein synthesis is first detected when oocytes enter their two week growth phase. Investigations in a number of species have concluded that the zona pellucida proteins are synthesized in oocytes and secreted into the zona matrix, although some studies have also identified zona proteins in granulosa cells. More recently, transcripts of the single copy mouse $Z p-2$ (15) and $Z p-3$ (16) genes have been detected only in growing oocytes and the biosynthesis of the ZP2 and ZP3 proteins is, likewise, restricted to oocytes $(17,18)$. At its peak in $30-35-\mu \mathrm{m}$ diameter oocytes, zona protein synthesis represents $7-8 \%$ of total protein synthesis. Zona production declines in fully grown oocytes and is absent in ovulated eggs. Once incorporated into the extracellular matrix, the zona pellucida proteins have a long half-life $(>100 \mathrm{~h})(19,20)$.
The biosynthetic pathways of ZP2 and ZP3 have been investigated using radioactive precursors and in vitro culture. The ZP2 core protein has a molecular weight of 76,373 , deduced from its nucleic acid sequence (15). Six complex-type $\mathrm{N}$-linked side chains and an undetermined number of O-linked oligosaccharides are attached to the core protein and the mature 120-140-kD glycoprotein is secreted and incorporated into the extracellular zona pellucida (21). The ZP3 protein is first detected in the oocyte as a 44,000-D protein to which three or four complex-type $\mathrm{N}$-linked carbohydrate side chains are added. The further attachment of O-linked sugars results in the production of the mature secreted $83-\mathrm{kD}$ ZP3 glycoprotein (18). The intracellular transport patterns and mechanisms of secretion for these proteins have yet to be delineated.

\section{Zona pellucida genes}

Structure of the mouse genes. Mouse $Z p-2$ and $Z p-3$, each single copy genes, are located on chromosomes 7 and 5, respectively (22). Mouse $Z p$ - 2 contains 18 exons (Fig. 2) that range in size from 45 to $190 \mathrm{bp}$ and are separated by 17 introns (81 to $1,490 \mathrm{bp}$ ). The gene spans $12.1 \mathrm{kbp}$ of DNA. Zp-2 is transcribed and processed into a 2,201-nt mRNA with very short $5^{\prime}$ $(30 \mathrm{nt})$ and $3^{\prime}(32 \mathrm{nt})$ untranslated regions. The detection of a 2.4-kb transcript in oocyte RNA suggests that ZP2 mRNA contains a poly(A) tail of $\sim 200 \mathrm{nt}$. ZP2 mRNA has a single open reading frame initiated at an ATG that encodes a polypeptide of 713 amino acids with a molecular weight of 80,217 . The first 34 amino acids represent a signal peptide that directs secretion and, following cleavage, the resultant core polypeptide $(76,373$ D) is incorporated into the extracellular matrix. The ZP2 amino acid sequence contains seven possible $\mathrm{N}$-linked glycosylation sites (Asn-X-Ser/Thr) and more than 100 potential Olinked glycosylation sites (15).

The 8.6-kbp mouse $Z p-3$ gene is comprised of eight exons (Fig. 2) ranging in size from 92 to $338 \mathrm{bp}$ and has introns whose lengths are between 125 and 2,320 bp (23). The 1,317-nt ZP3 mRNA has short $5^{\prime}(29 \mathrm{nt})$ and $3^{\prime}(16 \mathrm{nt})$ untranslated regions. The latter is so abbreviated that the TAA termination codon is 
A

Mouse $Z p-2$ Gene

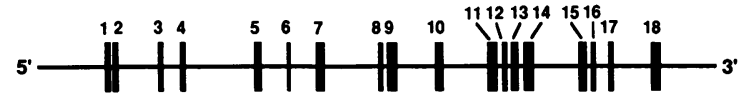

Human ZP2 Gene
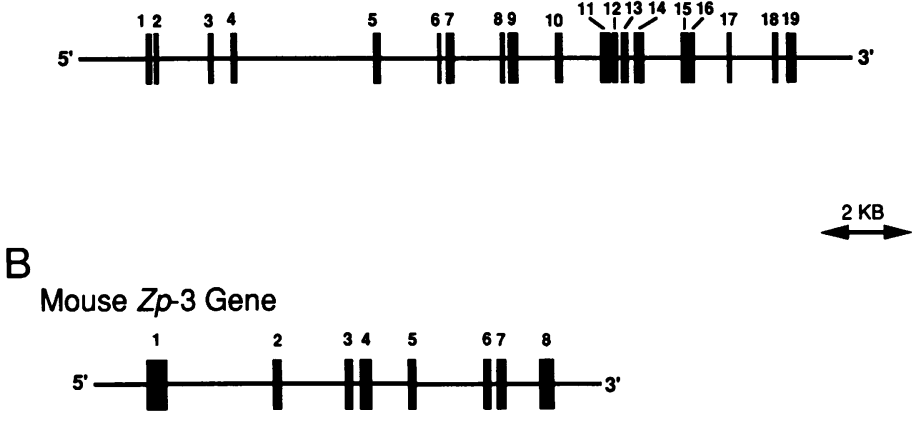

Figure 2. Genomic loci of human and mouse $Z p-2$ and $Z p-3$. (A) Comparison of mouse $Z p-2$ (18 exons) and human $Z P 2$ (19 exons) genes with transcription units of 12.1 and $13 \mathrm{kbp}$, respectively (15; and Liang, L. F., and J. Dean, manuscript in preparation). ( $B$ ) Alignment of

Human ZP3 Gene

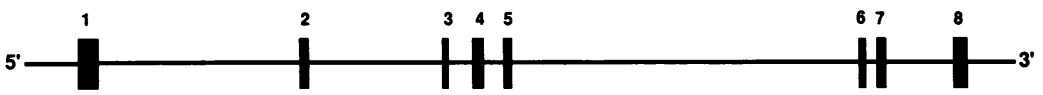
mouse $Z p-3(8.6 \mathrm{kbp})$ and human $Z P 3$ genes $(18.3 \mathrm{kbp})$, each of which contain eight exons $(23,25)$

embedded within the consensus AATAAA polyadenylation signal. Short untranslated regions are characteristic of both ZP2 (mouse and human) and ZP3 (mouse, human, and hamster) mRNAs. It is not clear what role, if any, these short untranslated regions play in gene expression, nor whether they are important for processing ZP2 and ZP3 transcripts. The polypeptide deduced from the single open reading frame of mouse ZP3 mRNA has a molecular weight of 46,307 and consists of 424 amino acids (24). The release of a predicted 22-amino acid signal peptide would result in the secretion of a 43,943-D protein, consistent with the reported 44-kD ZP3 core protein (18).

Although there is no overall similarity in the amino acid sequences of ZP2 and ZP3, they share at least one common structural motif. Each has a very hydrophobic region consisting of 23 and 26 amino acids, respectively, near the carboxyl terminus $(15,24)$. Hydrophobic domains like these are typically present in membrane-spanning domains of proteins. The hydrophobic regions in ZP2 and ZP3 may play an important role in the intracellular trafficking of these secreted proteins or in their interactions in the extracellular matrix. The carboxyl terminal hydrophobic domain is present in mouse (ZP2 and ZP3), human (ZP2 and ZP3), hamster (ZP3), and a recently described rabbit zona protein (15, 24-27; and Liang, L. F., and J. Dean, manuscript in preparation).

Conservation of the zona genes. The genes encoding $\mathrm{ZP} 2$ and $\mathrm{ZP} 3$ are conserved among mammals. Using cross-hybridization with mouse nucleic acid sequences as a criterion, the degree of conservation of $Z p-3$ is variable with the rat, dog, cow, and human zona genes being more related to mouse than the pig and rabbit genes (28). The human homologue of $\mathrm{Zp}-2$ and the human and hamster homologues of $Z p-3$ have been characterized. The human $Z P 2$ gene (Fig. 2) is composed of 19 exons (one more than the mouse) whose nucleic acid sequence is $70 \%$ the same as that of its mouse counterpart (Liang, L. F., and J. Dean, manuscript in preparation). The encoded proteins are $60 \%$ identical, although the human protein has 745 amino acids compared with 713 amino acids in mouse ZP2. The mouse, human, and hamster ZP3 genes contain eight exons each. The coding sequences of the mouse and human genes are $74 \%$ the same, and they encode 424 amino acid peptides that are $67 \%$ identical (25). The hamster gene encodes a 422-amino acid protein that is $81 \%$ identical to mouse ZP3 (26). The ZP3 mRNA in mouse oocytes is $1.5 \mathrm{~kb}$ (including a 200-nt poly(A) tail) and is indistinguishable in size from that of rat and rabbit ZP3 mRNAs (24). Taken together, these data suggest that the overall structure of the zona genes and their gene products are conserved among mammals.

\section{Expression of the zona genes}

Neither ZP2 nor ZP3 transcripts are present in resting mouse oocytes $(10-15 \mu \mathrm{m})$. However, once oocyte growth commences, increasing amounts of ZP2 and ZP3 transcripts are detected. Maximum levels of the transcripts (1,000 fg of ZP2 and $400 \mathrm{fg}$ of ZP3) are present in oocytes that are $50 \mu \mathrm{m}$ in diameter, representing 1 and $0.4 \%$, respectively, of total poly(A) ${ }^{+}$RNA. The molar ratio of ZP2 and ZP3 transcripts is $\sim 2: 1$, and this ratio is maintained during the growth phase of oocyte development $(15,16)$. As the oocyte reaches its full size (75-80 $\mu \mathrm{m})$, the amount of ZP2 and ZP3 transcripts declines, and, in ovulated eggs, the amount of these two transcripts is $<5 \%$ of its peak level (15). ZP2 and ZP3 mRNAs in ovulated eggs are $\sim 200 \mathrm{nt}$ shorter than they are in growing oocytes. This decrease corresponds to the estimated length of their poly(A) tails (15) and is very similar to the decrease observed for $\beta$-actin and $\alpha$-tubulin mRNAs in mouse eggs (29). This has led to the hypothesis that the zona mRNAs, like other maternal messages, undergo rapid deadenylation and degradation during meiotic maturation and ovulation.

Mechanism of oocyte-specific expression. The expression of mouse $Z p-2$ and $Z p-3$ is restricted to growing oocytes and is not detected in other mouse tissues $(15,16,24,28)$. The coordinate transcription of the zona genes is restricted to the two-week growth phase of oogenesis and serves both as a molecular marker for oocyte growth and differentiation, and as a paradigm for investigating molecular mechanisms of gene activation in the oocyte. Virtually nothing is known about the mecha- 
nisms that restrict gene expression to the female germline, and, although several previously characterized transcription factors are present in oocytes, their target genes have yet to be determined.

If the regulation of zona gene expression is under the control of shared regulatory factors, the DNA-binding sites of these factors may be evolutionarily conserved among mammals. A comparison of the first $300 \mathrm{bp}$ upstream of the initiation site of mouse and human $Z P 2$ genes reveals that they are $70 \%$ identical. There is a TATAA box at position $-31 \mathrm{bp}$ and a CCAAT box at position $-65 \mathrm{bp}$ in the mouse gene; these elements are present at comparable positions in the human gene (15; and Liang, L. F., and J. Dean, manuscript in preparation). In contrast, comparison of the $5^{\prime}$ flanking sequences of the mouse and human $Z P 3$ genes indicates no long stretches of sequence identity. The TATAA box at -29 bp in the mouse $Z p-3$ is also present in the human homologue. Neither $Z P 3$ gene has an identifiable CCAAT box $(23,25)$.

Interestingly, close examination of the first $300 \mathrm{bp}$ upstream of four zona genes (mouse $Z p-2$ and $Z p$-3; human $Z P 2$ and $Z P 3)$ revealed five short (4-12 bp), conserved DNA sequences (elements I, IIA, IIB, III, and IV), located at compardble distances from the transcription initiation site (30). We have demonstrated that one of these sequences, element IV (positioned $\sim 200$ bp upstream from the TATAA box), is necessary and sufficient for reporter gene expression driven by mouse $Z p-2$ and $Z p-3$ promoters microinjected into oocytes (30). Oligonucleotides containing element IV from either $Z p-2$ or Zp-3 form DNA-protein complexes of identical mobility in gel retardation assays using extracts of oocytes but not other tissues (Fig. 3).

The center of element IV from both the human and mouse $Z p-2$ and $Z p-3$ genes contains the motif CANNTG which has been identified as a consensus sequence (31) for the binding of basic helix-loop-helix (HLH) transcription factors (32). These factors generally bind DNA as homo- or heterodimers. The HLH domain is required for the protein-protein interactions involved in dimerization, while the adjacent basic domain interacts directly with DNA. HLH transcription factors have been shown to be involved in the regulation of transcription of several tissue specific genes (for review, see [33]).

The transcription factor-DNA complex identified in these experiments may contain one or more previously characterized basic HLH proteins, or may represent the first known example of an oocyte-specific transcription factor. The isolation and biochemical characterization of the zona gene activation factors will not only provide information about their structures, but will permit investigation of their putative functions as regulators of zona pellucida gene transcription in mouse oocytes. The concept of a common transcription factor(s) binding to element IV of the promoter region of the mouse $Z p-2$ and $Z p-3$ genes that participates in the coordinate and tissuespecific expression of the zona pellucida genes is an appealing hypothesis which can now be tested. The relatively low level of reporter gene activity in these studies (30) as well as in transgenic mice (34) suggests that additional regulatory factors may be important for in vivo levels of zona gene activity.

1. Abbreviation used in this paper: HLH, helix-loop-helix.

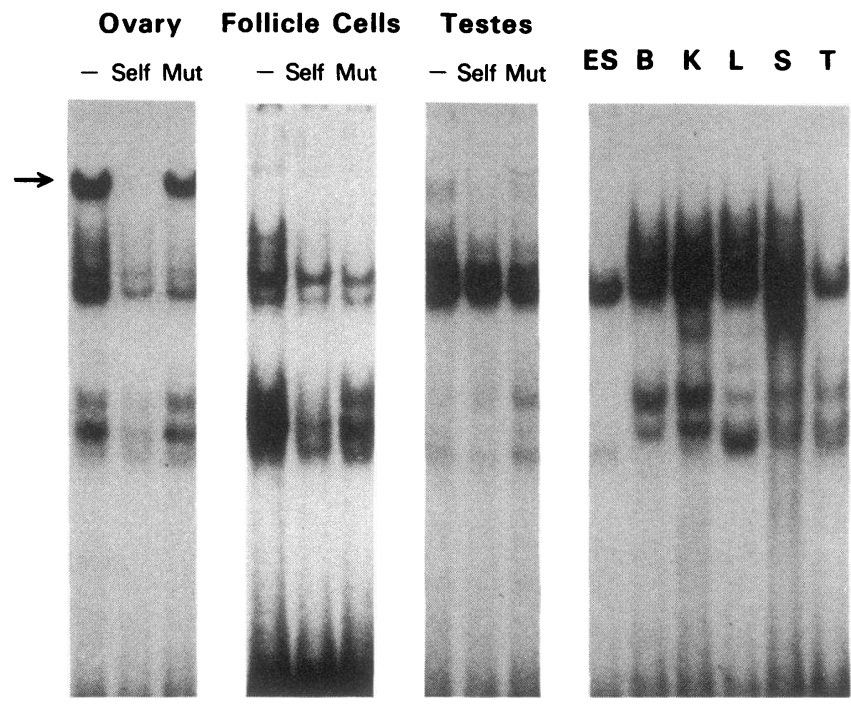

Figure 3. Ovary-specific binding of a transcription factor to a zona promoter element. Gel mobility shift assays using radioactively labelled oligonucleotides containing element IV from mouse $Z p-2$. Arrow indicates the oocyte-specific DNA-protein complex present in the direct binding assays with both $Z p-2$ and $Z p-3$ element IV oligonucleotides (30). Ovary, lane 1 , mouse $\left[{ }^{32} \mathrm{P}\right] Z p$ - 2 oligonucleotide containing element IV reacted with 2-3 $\mu \mathrm{g}$ of ovarian extract; lane 2, as lane 1 but competed with 50 -fold molar excess of unlabeled mouse $\mathrm{Zp}-2$ oligonucleotide; lane 3 , as lane 2 but competed with a $Z p-2$ oligonucleotide containing a 6-bp mutation in element IV. Follicle cells, as above but with 2-3 $\mu \mathrm{g}$ follicle cell protein. Testes, as above but with $2-3 \mu \mathrm{g}$ testes protein. Tissues, lanes $1-6$, mouse $\left[{ }^{32} \mathrm{P}\right] \mathrm{Zp}-2$ oligonucleotide reacted with 2-3 $\mu \mathrm{g}$ extract of embryonic stem cells (lane 1), brain (lane 2), kidney (lane 3), liver (lane 4), spleen (lane 5), and thymus (lane 6).

\section{Conclusions}

The elucidation of the primary structure of the zona proteins has provided a means for determining the protein and carbohydrate domains that are critical for species-specific fertilization. Genetic defects in the zona genes are expected to perturb female (but not male) gamete maturation, folliculogenesis, and fertilization. The establishment of animal models with null mutations in the zona genes (using embryonic stem cell technology) will define the phenotypes of such genetic defects and facilitate the search for their human counterparts. The oocytespecific zona pellucida promoters can be used to establish transgenic mouse lines expressing human zona pellucida genes. If the human $Z P 3$ (or $Z P 2$ ) transgene product is incorporated into the mouse zona pellucida, the transgenic animal could provide a much needed source of oocytes for assaying human sperm function in infertility clinics.

The zona pellucida has also been recognized as a target for immunocontraception. Passive administration of antibodies that bind to the zona pellucida prevent sperm penetration (35) and vaccination of mice with "self" zona peptides elicits antibodies that bind to the zona pellucida to cause long-term, reversible contraception (36). However, vaccination with zona pellucida peptides can also induce a $\mathrm{T}$ cell-mediated oophoritis in susceptible strains of mice (37), which have provided a model for investigating the pathogenesis of human autoimmune oophoritis. The precise definition of immunological re- 
sponse to different regions of the zona proteins is imperative in the rational design of contraceptive vaccines that will selectively induce antibodies leading to infertility, but not cause $T$ cell-mediated ovarian damage.

\section{References}

1. Hartmann, J. F., R. B. Gwatkin, and C. F. Hutchison. 1972. Early contact interactions between mammalian gametes in vitro: evidence that the vitellus influences adherence between sperm and zona pellucida. Proc. Natl. Acad. Sci. USA. 69:2767-2769.

2. Saling, P. M., J. Sowinski, and B. T. Storey. 1979. An ultrastructural study of epididymal mouse spermatozoa binding to zonae pellucidae in vitro: sequential relationship to the acrosome reaction. J. Exp. Zool. 209:229-238.

3. Bleil, J. D., and P. M. Wassarman. 1980. Mammalian sperm-egg interaction: identification of a glycoprotein in mouse egg zonae pellucidae possessing receptor activity for sperm. Cell. 20:873-882.

4. Florman, H. M., and P. M. Wassarman. 1985. O-linked oligosaccharides of mouse egg ZP3 account for its sperm receptor activity. Cell. 41:313-324.

5. Bleil, J. D., J. M. Greve, and P. M. Wassarman. 1988. Identification of a secondary sperm receptor in the mouse egg zona pellucida: role in maintenance of binding of acrosome-reacted sperm to eggs. Dev. Biol. 128:376-385.

6. Leyton, L., and P. Saling. 1989. Evidence that aggregation of mouse sperm receptors by ZP3 triggers the acrosome reaction. J. Cell Biol. 108:2163-2168.

7. Leyton, L., and P. Saling. $1989.95 \mathrm{kd}$ sperm proteins bind ZP3 and serve as tyrosine kinase substrates in response to zona binding. Cell. 57:1123-1130.

8. Bleil, J. D., and P. M. Wassarman. 1983. Sperm-egg interactions in the mouse: sequence of events and induction of the acrosome reaction by a zona pellucida glycoprotein. Dev. Biol. 95:317-324.

9. Saling, P. M. 1989. Mammalian sperm interaction with extracellular matrices of the egg. Oxf. Rev. Reprod. Biol. 11:339-388.

10. Sato, K. 1979. Polyspermy-preventing mechanisms in mouse eggs fertilized in vitro. J. Exp. Zool. 210:353-359.

11. Bleil, J. D., C. F. Beall, and P. M. Wassarman. 1981. Mammalian spermegg interaction: fertilization of mouse eggs triggers modification of the major zona pellucida glycoprotein, ZP2. Dev. Biol. 86:189-197.

12. Bleil, J. D., and P. M. Wassarman. 1988. Galactose at the nonreducing terminus of O-linked oligosaccharides of mouse egg zona pellucida glycoprotein ZP3 is essential for the glycoprotein's sperm receptor activity. Proc. Natl. Acad. Sci. USA. 85:6778-6782.

13. Shabanowitz, R. B., and M. G. O'Rand. 1988. Characterization of the human zona pellucida from fertilized and unfertilized eggs. J. Reprod. Fertil. 82:151-161.

14. Shabanowitz, R. B. 1990. Mouse antibodies to human zona pellucida: evidence that human $\mathrm{ZP3}$ is strongly immunogenic and contains two distinct isomer chains. Biol. Reprod. 43:260-270.

15. Liang, L.-F., S. M. Chamow, and J. Dean. 1990. Oocyte-specific expression of mouse $\mathrm{Zp}-2$ : developmental regulation of the zona pellucida genes. $\mathrm{Mol}$. Cell. Biol. 10:1507-1515.

16. Philpott, C. C., M. J. Ringuette, and J. Dean. 1987. Oocyte-specific expression and developmental regulation of $\mathrm{ZP} 3$, the sperm receptor of the mouse zona pellucida. Dev. Biol. 121:568-575.

17. Bleil, J. D., and P. M. Wassarman. 1980. Synthesis of zona pellucida proteins by denuded and follicle-enclosed mouse oocytes during culture in vitro. Proc. Natl. Acad. Sci. USA. 77:1029-1033.

18. Salzmann, G. S., J. M. Greve, R. J. Roller, and P. M. Wassarman. 1983. Biosynthesis of the sperm receptor during oogenesis in the mouse. EMBO (Eur. Mol. Biol. Organ.) J. 2:1451-1456.
19. Bleil, J. D., and P. M. Wassarman. 1980. Structure and function of the zona pellucida: identification and characterization of the proteins of the mouse oocyte's zona pellucida. Dev. Biol. 76:185-202.

20. Shimizu, S., M. Tsuji, and J. Dean. 1983. In vitro biosynthesis of three sulfated glycoproteins of murine zonae pellucidae by oocytes grown in follicle culture. J. Biol. Chem. 258:5858-5863.

21. Greve, J. M., G. S. Salzmann, R. J. Roller, and P. M. Wassarman. 1982. Biosynthesis of the major zona pellucida glycoprotein secreted by oocytes during mammalian oogenesis. Cell. 31:749-759.

22. Lunsford, R. D., N. A. Jenkins, C. A. Kozak, L.-F. Liang, C. M. Silan, N. G. Copeland, and J. Dean. 1990. Genomic mapping of murine Zp-2 and Zp-3, two oocyte-specific loci encoding zona pellucida proteins. Genomics. 6:184-187.

23. Chamberlin, M. E., and J. Dean. 1989. Genomic organization of a sex specific gene: the primary sperm receptor of the mouse zona pellucida. Dev. Biol. 131:207-214.

24. Ringuette, M. J., M. E. Chamberlin, A. W. Baur, D. A. Sobieski, and J. Dean. 1988. Molecular analysis of cDNA coding for ZP3, a sperm binding protein of the mouse zona pellucida. Dev. Biol. 127:287-295.

25. Chamberlin, M. E., and J. Dean. 1990. Human homolog of the mouse sperm receptor. Proc. Natl. Acad. Sci. USA. 87:6014-6018.

26. Kinloch, R. A., B. Ruiz-Seiler, and P. M. Wassarman. 1990. Genomic organization and polypeptide primary structure of zona pellucida glycoprotein hZP3, the hamster sperm receptor. Dev. Biol. 142:414-421.

27. Schwoebel, E., S. Prasad, T. M. Timmons, R. Cook, H. Kimura, E.-M. Niu, P. Cheung, S. Skinner, S. E. Avery, B. Wilkins, and B. S. Dunbar. 1991. Isolation and characterization of a full-length cDNA encoding the $55 \mathrm{kDa}$ rabbit zona pellucida protein. J. Biol. Chem. 266:7214-7219.

28. Ringuette, M. J., D. A. Sobieski, S. M. Chamow, and J. Dean. 1986. Oocyte-specific gene expression: molecular characterization of a cDNA coding for ZP-3, the sperm receptor of the mouse zona pellucida. Proc. Natl. Acad. Sci. USA. 83:4341-4345.

29. Paynton, B. V., R. Rempel, and R. Bachvarova. 1988. Changes in state of adenylation and time course of degradation of maternal mRNAs during oocyte maturation and early embryonic development in the mouse. Dev. Biol. 129:304314.

30. Millar, S. E., E. Lader, L.-F. Liang, and J. Dean. 1991. Oocyte-specific factors bind a conserved upstream sequence required for mouse zona pellucida promoter activity. Mol. Cell. Biol. 12:6197-6204.

31. Lassar, A. B., J. N. Buskin, D. Lockshon, R. L. Davis, S. Apone, S. D. Hauschka, and H. Weintraub. 1989. MyoD is a sequence-specific DNA binding protein requiring a region of myc homology to bind to the muscle creatine kinase enhancer. Cell. 58:823-831.

32. Murre, C., P. S. McCaw, and D. Baltimore. 1989. A new DNA binding and dimerization motif in immunoglobulin enhancer binding, daughterless, MyoD, and myc proteins. Cell. 56:777-783.

33. Blackwell, T. K., and H. Weintraub. 1990. Differences and similarities in DNA-binding preferences of myoD and E2A protein complexes revealed by binding site selection. Science (Wash. DC). 250:1104-1110.

34. Lira, S. A., R. A. Kinloch, S. Mortillo, and P. M. Wassarman. 1990. An upstream region of the mouse $\mathrm{ZP3}$ gene directs expression of firefly luciferase specifically to growing oocytes in transgenic mice. Proc. Natl. Acad. Sci. USA. 87:7215-7219.

35. East, I. J., B. J. Gulyas, and J. Dean. 1985. Monoclonal antibodies to the murine zona pellucida protein with sperm receptor activity: effects on fertilization and early development. Dev. Biol. 109:268-273.

36. Millar, S. E., S. M. Chamow, A. W. Baur, C. Oliver, F. Robey, and J. Dean. 1989. Vaccination with a synthetic zona pellucida peptide produces longterm contraception in female mice. Science (Wash. DC). 246:935-938.

37. Rhim, S. H., S. E. Millar, F. Robey, A.-M. Luo, Y.-H. Lou, P. Allen, J. Dean, and K. S. K. Tung. 1992. Autoimmune disease of the ovary induced by a ZP3 peptide from the mouse zona pellucida. J. Clin. Invest. 89:28-35. 